Bian W, French M (2005) Graph topologies, gap metrics, and robust stability for nonlinear systems. SIAM J Control Optim 44:418-443

El-Sakkary AK (1985) The gap metric: robustness of stabilization of feedback systems. IEEE Trans Autom Control 30:240-247

Feintuch A (1998) Robust control theory in Hilbert space. Springer, New York

Foias C, Georgiou TT, Smith MC (1993) Robust stability of feedback systems: a geometric approach using the gap metric. SIAM J Control Optim 31:1518-1537

Georgiou TT (1988) On the computation of the gap metric. Syst Control Lett 11:253-257

Georgiou TT, Smith MC (1990) Optimal robustness in the gap metric. IEEE Trans Autom Control 35:673-687

Georgiou TT, Smith MC (1992) Robust stabilization in the gap metric: controller design for distributed plants. IEEE Trans Autom Control 37:1133-1143

Georgiou TT, Smith MC (1997) Robustness analysis of nonlinear feedback systems: an input-output approach. IEEE Trans Autom Control 42:1200-1221

Glover K, McFarlane DC (1989) Robust stabilization of normalized coprime factor plant descriptions with $\mathcal{H}_{\infty}$ bounded uncertainties. IEEE Trans Autom Control 34:821-830

James MR, Smith MC, Vinnicombe G (2005) Gap metrics, representations and nonlinear robust stability. SIAM J Control Optim 43:1535-1582

Kato T (1976) Perturbation theory for linear operators, 2nd edn. Springer, Berlin

McFarlane DC, Glover K (1992) A loop shaping design procedure using $\mathcal{H}_{\infty}$-synthesis. IEEE Trans Autom Control 37:759-769

Qiu L, Davison EJ (1992a) Feedback stability under simultaneous gap metric uncertainties in plant and controller. Syst Control Lett 18:9-22

Qiu L, Davison EJ (1992b) Pointwise gap metrics on transfer matrices. IEEE Trans Autom Control 37:741-758

Qiu L, Zhang Y, Li CK (2008) Unitarily invariant metrics on the Grassmann space. SIAM J Matrix Anal 27:501-531

Qiu L, Zhou K (2013) Preclassical tools for postmodern control. IEEE Control Syst Mag 33(4): 26-38

Vidyasagar M (1984) The graph metric for unstable plants and robustness estimates for feedback stability. IEEE Trans Autom Control 29:403-418

Vidyasagar M (1985) Control system synthesis: a factorization approach. MIT, Cambridge

Vinnicombe G (1993) Frequency domain uncertainty and the graph topology. IEEE Trans Autom Control 38:1371-1383

Vinnicombe G (2001) Uncertainty and feedback: $\mathcal{H}_{\infty}$ loop-shaping and the $v$-gap metric. Imperial Collage Press, London

Zames G, El-Sakkary AK (1980) Unstable systems and feedback: the gap metric. In: Proceedings of the 16th Allerton conference, Illinois, pp 380-385

Zhang Y, Qiu L (2010) From subadditive inequalities of singular values to triangular inequalities of canonical angles. SIAM J Matrix Anal Appl 31:1606-1620
Zhou K, Doyle JC (1998) Essentials of robust control. Prentice Hall, Upper Saddle River

\section{Robust Control of Infinite Dimensional Systems}

\author{
Hitay Özbay \\ Department of Electrical and Electronics \\ Engineering, Bilkent University, Ankara, Turkey
}

\section{Abstract}

Basic robust control problems are studied for the feedback systems where the underlying plant model is infinite dimensional. The $\mathcal{H}_{\infty}$ optimal controller formula is given for the mixed sensitivity minimization problem with rational weights. Key steps of the numerical computations required to determine the controller parameters are illustrated with an example where the plant model include time delay terms.

\section{Keywords}

Coprime factorizations; Direct design methods; Inner-outer factorizations

\section{Introduction}

Robust control deals with the feedback system shown in Fig. 1, where $P_{\Delta}$ represents the uncertain physical plant and $C$ is a fixed controller to be designed.

Here, it is assumed that the controller and the plant are linear time invariant (LTI) systems and

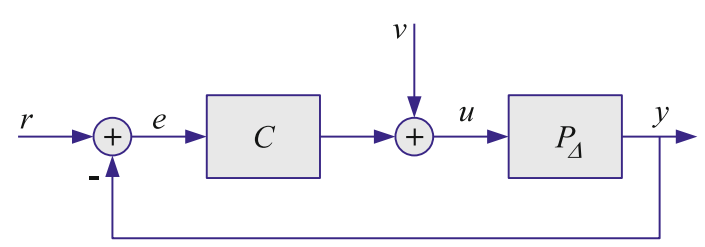

Robust Control of Infinite Dimensional Systems, Fig. 1 Feedback system $\mathcal{F}\left(C, P_{\Delta}\right)$ with fixed controller $C$ and uncertain plant $P_{\Delta}$ 
they are represented by their transfer functions. Furthermore, $P_{\Delta}$ satisfies the following conditions:

$$
P_{\Delta}(s)=P(s)+\Delta(s)
$$

where $P$ is the nominal plant model, with $P(s)$ and $P_{\Delta}(s)$ having the same number of poles in $\mathbb{C}_{+} ;$and there is a known uncertainty bound $W(s)$ satisfying

$$
|\Delta(j \omega)|<|W(j \omega)| \quad \forall \omega \in \mathbb{R} .
$$

Definition 1 All $P_{\Delta}$ satisfying the above conditions are said to be in the set of uncertain plants $\mathcal{P}$, which is characterized by the given functions $P(s)$ and $W(s)$.

Depending on physical system modeling, other forms of uncertainty representations can be more convenient than the additive unstructured uncertainty model taken here; see, e.g., Doyle et al. (1992), Özbay (2000), and Zhou et al. (1996) for the examples of multiplicative, coprime factor, parametric, and structured uncertainty descriptions. Note that for notational convenience and simplicity of the presentation, single-input-single-output (SISO) plants are considered here; for extensions to multi-inputmulti-output (MIMO) plants, see, e.g., Curtain and Zwart (1995).

When the plant under consideration is infinite dimensional, the transfer function $P(s)$ is irrational, i.e., it cannot be expressed as a ratio of two polynomials (it does not admit a finitedimensional state-space representation). Typical examples of such systems are spatially distributed parameter systems modeled by partial differential equations, fractional-order systems, and systems with time delays. The reader is referred to Curtain and Morris (2009) for examples of transfer functions of distributed parameter systems. There are many interesting industrial applications where fractional-order transfer functions are used for modeling and control, see, e.g., Monje et al. (2010); typically, such functions are rational in $s^{\alpha}$, where $\alpha$ is a rational number in the open interval $(0,1)$. Transfer functions of systems with time delays involve terms like $e^{-h s}$ where $h>0$ is the delay; see Sipahi et al. (2011) for various real-life examples where time-delay models appear. Transfer functions considered here are functions of the complex variable $s$ with real coefficients, so $\overline{P(\bar{s})}=P(s)$ where $\bar{s}$ denotes the complex conjugate of $s$.

Definition 2 A linear time invariant system $H$ is said to be stable if its transfer function $H(s)$ is bounded and analytic in $\mathbb{C}_{+}$. In this case, the system norm is

$$
\|H\|=\|H\|_{\infty}=\sup _{\operatorname{Re}(s)>0}|H(s)|,
$$

which is equivalent to the energy amplification through the system $H$; see Doyle et al. (1992) and Foias et al. (1996).

Definition 2 is sometimes called the $\mathcal{H}_{\infty^{-}}$ stability, and in this setting, the set of all stable plants is the function space $\mathcal{H}_{\infty}$. It is worth noting that for infinite-dimensional systems, there are other definitions of stability (Curtain and Zwart 1995; Desoer and Vidyasagar 2009), leading to different measures of the system norm.

\section{Robust Control Design Objectives}

Let $\mathcal{F}\left(C, P_{\Delta}\right)$ denote the feedback system shown in Fig. 1. This system is said to be robustly stable if all the transfer functions from external inputs $(r, v)$ to internal signals $(e, u)$ are in $\mathcal{H}_{\infty}$ for all $P_{\Delta} \in \mathcal{P}$. In the controller design, robust stability of the feedback system is the primary constraint.

The feedback system $\mathcal{F}\left(C, P_{\Delta}\right)$ is robustly stable if and only if the following conditions hold; see, e.g., Doyle et al. (1992) and Foias et al. (1996),

(a) $S, C S, P S \in \mathcal{H}_{\infty}$, where $S=(1+P C)^{-1}$, and

(b) $\|\mathrm{W} \mathrm{CS}\|_{\infty} \leq 1$.

In order to illustrate these design constraints for robustly stabilizing controller, as an example, consider a strictly proper stable plant, i.e.,

$$
P \in \mathcal{H}_{\infty} \quad \text { with } \quad \lim _{|s| \rightarrow \infty}|P(s)|=0 .
$$


In this case, all controllers in the form $C=$ $Q /(1-P Q)$ satisfy condition (a) for any $Q \in$ $\mathcal{H}_{\infty}$ (moreover, any controller $C$ satisfying $(a)$ must be in this form for some $Q \in \mathcal{H}_{\infty}$ ). Now consider a rational $W(s)$ with a stable $Q$ such that $|Q(j \omega)|$ is a continuous function of $\omega \in \mathbb{R}$. Then, condition $(b)$ becomes

$$
\begin{gathered}
\|W Q\|_{\infty} \leq 1 \Longleftrightarrow|Q(j \omega)| \leq|W(j \omega)|^{-1} \\
\forall \omega \in \mathbb{R} .
\end{gathered}
$$

So, whenever the modeling uncertainty is "large" on a frequency band $\omega \in \Omega$, the magnitude of $Q$ should be "small" in this region.

When the plant is unstable, say $p \in \mathbb{C}_{+}$is a pole of $P(s)$ of multiplicity one, conditions $(a)$ and $(b)$ impose a restriction on the controller, that leads to

$$
\begin{aligned}
1 & \geq\|\mathrm{WCS}\|_{\infty} \geq\left|\frac{W(p)}{N(p)}\right| \text { where } N(p) \\
& =\lim _{s \rightarrow p} \frac{(s-p)}{(s+\bar{p})} P(s) .
\end{aligned}
$$

So, a necessary condition, for $(b)$ to hold in this case, is $|W(p)| \leq|N(p)|$, which means that the modeling uncertainty at the unstable pole of the plant should be small enough for the existence of a robustly stabilizing controller. This is one of the fundamental quantifiable limitations of feedback systems with unstable plants; see Stein (2003) for further discussions on other limitations.

Many other performance-related design objectives, such as reference tracking and disturbance attenuation, are captured by the sensitivity minimization, which is defined as finding a controller satisfying (a) and achieving

\section{(c) $\left\|W_{1} S\right\|_{\infty} \leq \gamma$}

for the smallest possible $\gamma>0$, for a given stable sensitivity weight $W_{1}(s)$. Selection of $W_{1}$ depends on the class of reference signals and disturbances considered; see Doyle et al. (1992), Özbay (2000), and Stein (2003) for general guidelines. Stability robustness and performance objectives defined above can be blended to define a single
$\mathcal{H}_{\infty}$-optimization problem, known as the mixed sensitivity minimization: given $W_{1}, W_{2}, P$, find a controller $C$ satisfying $(a)$ and achieving

$$
\begin{aligned}
& \text { (d) }\left\|\left[\begin{array}{l}
W_{1} S \\
W_{2} T
\end{array}\right]\right\|_{\infty}:= \\
& \sup _{\operatorname{Re}(s)>0}\left(\left|W_{1}(s) S(s)\right|^{2}+\left|W_{2}(s) T(s)\right|^{2}\right)^{\frac{1}{2}} \leq \gamma
\end{aligned}
$$

for the smallest possible $\gamma>0$, where $T(s):=1-S(s)$ and $W_{2}(s)$ represents the multiplicative uncertainty bound, with $\left|W_{2}(j \omega)\right|=|W(j \omega)| /|P(j \omega)|, \forall \omega \in \mathbb{R}$. The smallest achievable $\gamma$ is the optimal performance level $\gamma_{\text {opt }}$ and the corresponding controller is denoted by $C_{\text {opt }}$. Typically, when $P$ is infinite dimensional so is the optimal controller.

\section{Design Methods}

\section{Approximation of the Plant}

One possible way to design a robust controller for an infinite-dimensional plant $P$ is to design a robust controller $C_{a}$ for an approximate finitedimensional plant $P_{a}$; (for a frequency domain approximation technique for infinite-dimensional systems, see Gu et al. 1989). When $W_{1}, W_{2}$, and $P_{a}$ are finite dimensional, standard state-space methods, Zhou et al. (1996), can be used to find an $\mathcal{H}_{\infty}$ controller $C_{a}$ achieving

$$
\left\|\left[\begin{array}{l}
W_{1} S_{a} \\
W_{2} T_{a}
\end{array}\right]\right\|_{\infty} \leq \gamma_{a}
$$

for the smallest possible $\gamma_{a}$, where $S_{a}:=(1+$ $\left.P_{a} C_{a}\right)^{-1}$ and $T_{a}=\left(1-S_{a}\right)$. Then, the controller $C=C_{a}$ satisfies $(a)$ and achieves the performance objective $(d)$ with

$$
\gamma=\left(\gamma_{a}+\varepsilon\right) \frac{1}{1-\varepsilon}, \quad \varepsilon:=\left\|C_{a} S_{a}\left(P-P_{a}\right)\right\|_{\infty},
$$

where it is assumed that the approximation of the plant is made in such a way that $\varepsilon<1$. Clearly, if $\gamma_{a} \rightarrow \gamma_{\mathrm{opt}}$ as $\varepsilon \rightarrow 0$, then $\gamma \rightarrow \gamma_{\mathrm{opt}}$ as 
$\varepsilon \rightarrow 0$. The conditions under which $\gamma_{a} \rightarrow \gamma_{\mathrm{opt}}$ are discussed in Morris (2001).

\section{Direct Design Methods}

The classical two-Riccati equation approach, Zhou et al. (1996), developed for finitedimensional systems, has been extended to various classes of infinite-dimensional systems by using the state-space techniques where semigroup theory plays an important role; see van Keulen (1993) for further details.

In order to illustrate some of the key steps of a frequency domain method developed in Foias et al. (1996), consider a specific example where the plant is given as

$$
\begin{aligned}
P(s) & =\frac{(s-1)(s+2) e^{-h s}}{\left(s^{2}+2 s+2\right)\left(s+1-3 e^{-2 h s}\right)}, \\
h & =\ln (2) \approx 0.693 .
\end{aligned}
$$

First, compute the location of the poles in $\mathbb{C}_{+}$ using available numerical tools for finding the roots of quasi-polynomials; see, e.g., Sipahi et al. (2011) for references. For the simple example chosen here, $P(s)$ has only one pole in $\mathbb{C}_{+}$, at $s=0.5$ (for larger values of $h$, the number of unstable poles of $P$ may be higher). Now, the plant can be factored as follows:

$$
P(s)=\frac{M_{N}(s) N_{O}(s)}{M_{D}(s)}
$$

where

$$
M_{N}(s)=\frac{s-1}{s+1} e^{-h s} \quad M_{D}(s)=\frac{s-0.5}{s+0.5}
$$

are all-pass (inner) transfer functions and

$$
\begin{aligned}
N_{O}(s)= & \frac{(s+2)(s+1)}{\left(s^{2}+2 s^{2}+2\right)(s+0.5)} \\
& \left(\frac{s-0.5}{s+1-3 e^{-2 h s}}\right)
\end{aligned}
$$

is a minimum-phase (outer) transfer function. Note that

$$
\begin{aligned}
\frac{s-0.5}{s+1-3 e^{-2 h s}} & =\frac{1}{1+H_{F}(s)}, \\
H_{F}(s) & =1.5 \frac{1-e^{-2 h(s-0.5)}}{s-0.5} .
\end{aligned}
$$

The impulse response of $H_{F}$ is $h_{F}(t)=1.5 e^{t / 2}$ when $t \in[0,2 h]$ and $h_{F}(t)=0$ otherwise. Stability of $N_{O}$ can also be verified from the Nyquist graph of $H_{F}$. Also, note that $N_{O}(s)$ can be factored as $N_{O}(s)=N_{1}(s) N_{2}(s)$ where

$$
\begin{aligned}
& N_{1}(s)=\frac{(s+2)(s+1)}{\left(s^{2}+2 s^{2}+2\right)}\left(\frac{1}{1+H_{F}(s)}\right), \\
& N_{2}(s)=\frac{1}{s+0.5}
\end{aligned}
$$

with $N_{1}, N_{1}^{-1} \in \mathcal{H}_{\infty}$ and $N_{2}$ is finite-dimensional (first order in this example).

The above steps illustrate coprime factorizations and inner-outer factorizations for systems with time delays (retarded case). For systems represented by PDEs or integrodifferential equations, plant transfer function can be factored similarly, provided that the poles and zeros in $\mathbb{C}_{+}$can be computed numerically.

When the plant is in the form (2) given above and the weights $W_{1}$ and $W_{2}$ are rational, the optimal performance level and the corresponding optimal controller is obtained by the following procedure (see Foias et al. (1996) for details).

- Controller parameterization transforms the mixed sensitivity minimization to a problem of finding the smallest $\gamma>0$ for which there exists $Q \in \mathcal{H}_{\infty}$ such that

$\left\|\left[\begin{array}{c}W_{1} \\ 0\end{array}\right]-\left[\begin{array}{c}W_{1} N_{2} \\ -W_{2} N_{2}\end{array}\right] M_{N}\left(R+M_{D} Q\right)\right\|_{\infty} \leq \gamma$

where $R(s)$ is a rational function (whose order is one less than the order of $M_{D}$ ) satisfying certain interpolation conditions at the zeros of $M_{D}(s)$.

- A spectral factorization determines $W_{0} \in \mathcal{H}_{\infty}$ such that $W_{0}^{-1} \in \mathcal{H}_{\infty}$ and

$$
\begin{gathered}
\left(\left|W_{1}(j \omega)\right|^{2}+\left|W_{2}(j \omega)\right|^{2}\right)\left|N_{2}(j \omega)\right|^{2} \\
=\left|W_{0}(j \omega)\right|^{2} \quad \forall \omega \in \mathbb{R},
\end{gathered}
$$


(here, it is assumed that $W_{2} N_{2}$ and $\left(W_{2} N_{2}\right)^{-1}$ are in $\mathcal{H}_{\infty}$ ).

- By using the norm preserving property of the unitary matrices and the commutant lifting theorem, it has been shown that

$$
\gamma_{\mathrm{opt}}=\left\|\left[\begin{array}{l}
\Gamma \\
\Upsilon
\end{array}\right]\right\|
$$

where $\Gamma$ is the Hankel operator whose symbol is

$$
M_{D}(-s)\left(M_{N}(-s) W_{0}^{-1}(-s) N_{2}(-s) W_{1}(-s) W_{1}(s)-W_{0}(s) R(s)\right)
$$

and $\Upsilon$ is the Toeplitz operator whose symbol is $W_{1}(s) W_{2}(s) N_{2}(s) W_{0}^{-1}(s)$. Moreover, under mild technical assumptions, the optimal controller is obtained from a nonzero $\psi_{o} \in \mathcal{H}_{2}$ satisfying

$$
\left(\gamma_{\mathrm{opt}}^{2}-\left(\Gamma^{*} \Gamma+\Upsilon^{*} \Upsilon\right)\right) \psi_{o}=0
$$

The operator $\left(\Gamma^{*} \Gamma+\Upsilon^{*} \Upsilon\right)$ is in the form of a skew-Toeplitz operator that gives the name to this approach. See Foias et al. (1996) for a detailed exposition.

\section{Optimal $\mathcal{H}_{\infty}$-Controller}

The above steps have been implemented, and the final optimal controller expression has been obtained in a simplified form described below.

Let $\alpha_{1}, \ldots, \alpha_{\ell} \in \mathbb{C}_{+}$be the zeros of $M_{D}(s)$, i.e., unstable poles of the plant (for simplicity of the exposition, they are assumed to be distinct). The sensitivity weight can be written as $W_{1}(s)=n W_{1}(s) / d W_{1}(s)$, for two coprime polynomials $n W_{1}$ and $d W_{1}$ with $\operatorname{deg}\left(n W_{1}\right) \leq \operatorname{deg}\left(d W_{1}\right)=: n_{1} \geq 1$. Define

$$
E_{\gamma}(s):=\left(\frac{W_{1}(-s) W_{1}(s)}{\gamma^{2}}-1\right)
$$

and let $\beta_{1}, \ldots, \beta_{2 n_{1}}$ be the zeros of $E_{\gamma}(s)$, enumerated in such a way that $-\beta_{n_{1}+k}=$ $\beta_{k} \in \overline{\mathbb{C}}_{+}$, for $k=1, \ldots, n_{1}$. For notational convenience, assume that the zeros of $E_{\gamma}$ are distinct for $\gamma=\gamma_{\mathrm{opt}}$.

Now define a rational function depending on $\gamma>0$ and the weights $W_{1}$ and $W_{2}$,

$$
F_{\gamma}(s):=\gamma \frac{d W_{1}(-s)}{n W_{1}(s)} G_{\gamma}(s)
$$

where $G_{\gamma} \in \mathcal{H}_{\infty}$ is an outer function determined from the spectral factorization

$$
\begin{aligned}
& G_{\gamma}(-s) G_{\gamma}(s) \\
& \quad=\left(1+\frac{W_{2}(-s) W_{2}(s)}{W_{1}(-s) W_{1}(s)}-\frac{W_{2}(-s) W_{2}(s)}{\gamma^{2}}\right)^{-1} .
\end{aligned}
$$

With the above definitions, under certain mild conditions (satisfied generically in most practical cases), the optimal controller can be expressed as

$$
C_{\mathrm{opt}}(s)=\frac{E_{\gamma}(s) M_{D}(s) F_{\gamma}(s) L(s)}{1+M_{N}(s) F_{\gamma}(s) L(s)} N_{O}^{-1}(s)
$$

where $\gamma=\gamma_{\mathrm{opt}}$ and $L(s)=L_{2}(s) / L_{1}(s)$ with polynomials $L_{1}$ and $L_{2}$, of degree $n_{1}+\ell-1$, determined from the interpolation conditions:

$$
\begin{array}{rlrl}
L_{1}\left(\beta_{k}\right)+M_{N}\left(\beta_{k}\right) F_{\gamma}\left(\beta_{k}\right) L_{2}\left(\beta_{k}\right) & =0 & & k=1, \ldots, n_{1} \\
L_{1}\left(\alpha_{k}\right)+M_{N}\left(\alpha_{k}\right) F_{\gamma}\left(\alpha_{k}\right) L_{2}\left(\alpha_{k}\right) & =0 & k & =1, \ldots, \ell \\
L_{2}\left(-\beta_{k}\right)+M_{N}\left(\beta_{k}\right) F_{\gamma}\left(\beta_{k}\right) L_{1}\left(-\beta_{k}\right) & =0 & k & =1, \ldots, n_{1} \\
L_{2}\left(-\alpha_{k}\right)+M_{N}\left(\alpha_{k}\right) F_{\gamma}\left(\alpha_{k}\right) L_{1}\left(-\alpha_{k}\right) & =0 & k & =1, \ldots, \ell .
\end{array}
$$


The above system of equations can be rewritten in the matrix form

$$
\mathcal{R}_{\gamma} \Phi=0
$$

where the $2\left(n_{1}+\ell\right) \times 1$ vector $\Phi$ contains the coefficients of $L_{1}$ and $L_{2}$, and $\mathcal{R}_{\gamma}$ is a $2\left(n_{1}+\right.$ $\ell) \times 2\left(n_{1}+\ell\right)$ matrix which can be computed numerically when $\gamma$ is fixed. The optimal performance level $\gamma_{\text {opt }}$ is the largest $\gamma$ which makes $\mathcal{R}_{\gamma}$ singular. The corresponding nonzero $\Phi$ gives $L(s)$, and hence, all the components of $C_{\mathrm{opt}}$ are computed.

Example 1 Consider the weighted sensitivity minimization for the plant (1) with the following first-order weights:

$$
W_{1}(s)=\frac{1}{s}, \quad W_{2}(s)=k s
$$

where $k>0$ represents the relative importance of the multiplicative uncertainty with respect to the tracking performance under steplike reference inputs (see Doyle et al. 1992; Özbay 2000). With (6) the functions $E_{\gamma}(s)$ and $F_{\gamma}(s)$ are computed as

$$
\begin{aligned}
& E_{\gamma}(s)=\frac{1+\gamma^{2} s^{2}}{-\gamma^{2} s^{2}}, \quad F_{\gamma}(s)=\frac{-\gamma s}{k s^{2}+k_{\gamma} s+1}, \\
& \text { where } \quad k_{\gamma}=\sqrt{2 k-\frac{k^{2}}{\gamma^{2}}} .
\end{aligned}
$$

In this example $\ell=1$ and $n_{1}=1$, with $\alpha_{1}=$ $0.5, \beta_{1}=j / \gamma$. For $k=0.1$, the largest $\gamma$ which makes $\mathcal{R}_{\gamma}$ singular is $\gamma_{\text {opt }}=7.452$, and the coefficients of the corresponding $L(s)$ are computed from the SVD of $\mathcal{R}_{\gamma_{\text {opt }} \text {, }}$

$$
L(s)=\frac{-0.0867-0.99623 s}{-0.0867+0.99623 s}=\frac{0.087+s}{0.087-s} .
$$

Note that zeros of $E_{\gamma}(s) M_{D}(s)$ in $\overline{\mathbb{C}}_{+}$appear as roots of the equation

$$
1+M_{N}(s) F_{\gamma}(s) L(s)=0 .
$$

Hence, there are internal unstable pole-zero cancelations in the representation (4). An internally stable implementation of this controller is shown in Gumussoy (2011) using a realization similar to (3).

The above approach can also be extended to a class of infinite-dimensional plants with infinitely many poles in $\mathbb{C}_{+}$; see Gumussoy and Özbay (2004) for technical details.

\section{Summary and Future Directions}

This entry briefly summarized robust control problems involving linear time invariant infinitedimensional plants with dynamic uncertainty models. Salient features of these robust control problems are captured by the mixed sensitivity minimization problem, for which a numerical computational procedure is outlined under the assumption that the weights are rational functions. Note that different types of plant models involving probabilistic, parametric, or structured (MIMO case) uncertainty are left out in this entry. Other robust control problems that are not discussed here include simultaneous stabilization (control of finitely many plant models by a single robust controller) and strong stabilization (robust control with the added restriction that the controller must be stable) of infinite-dimensional systems. Stable robust controller design techniques for different types of systems with time delays are illustrated in Özbay (2010) and Wakaiki et al. (2013); see also their references.

For practical implementation of infinitedimensional robust controllers, it is important to find low-order approximations of stable irrational transfer functions with prescribed $\mathcal{H}_{\infty}$ error bound. There exist many different approximation techniques for various types of transfer functions, but there is still need for computationally efficient algorithms in this area. Another interesting topic along the same lines is direct computation of fixed-order $\mathcal{H}_{\infty}$ controllers for infinitedimensional plants. In fact, computation of $\mathcal{H}_{\infty}{ }^{-}$ optimal PID controllers is still a challenging 
problem for infinite-dimensional plants, except for some time-delay systems satisfying certain simplifying structural assumptions. Advances in numerical optimization tools will play critical roles in the computation of low (or fixed)-order robust controller design for infinite-dimensional plants; see, e.g., Gumussoy and Michiels (2011) for recent results along this direction.

In the past, robust control of infinitedimensional systems found applications in many different areas such as chemical processes, flexible structures, robotic systems, transportation systems, and aerospace. Robust control problems involving systems with timevarying and uncertain time delays appear in control of networks and control over networks. Ongoing research in the networked systems area include generalization of these problems to more complex and interconnected systems.

There are also many interesting robust control problems in biological systems, where typical underlying plant models are nonlinear and infinite dimensional. Some of these problems are solved under simplifying assumptions; it is expected that robust control theory will make significant contributions to this field by extensions of the existing results to more realistic plant and uncertainty models.

\section{Cross-References}

- Control of Linear Systems with Delays

- Flexible Robots

- H-Infinity Control

- Model Order Reduction: Techniques and Tools

- Networked Systems

- Optimal Control via Factorization and Model Matching

- Optimization Based Robust Control

$\checkmark$ PID Control

- Robust Control in Gap Metric

- Spectral Factorization

- Stability and Performance of Complex Systems Affected by Parametric Uncertainty

- Structured Singular Value and Applications: Analyzing the Effect of Linear Time-Invariant Uncertainty in Linear Systems

\section{Bibliography}

Curtain R, Morris K (2009) Transfer functions of distributed parameter systems: a tutorial. Automatica 45:1101-1116

Curtain R, Zwart HJ (1995) An introduction to infinite-dimensional linear systems theory. Springer, New York

Desoer CA, Vidyasagar M (2009) Feedback systems: input-output properties. SIAM, Philadelphia

Doyle JC, Francis BA, Tannenbaum AR (1992) Feedback control theory. Macmillan, New York

Foias C, Özbay H, Tannenbaum A (1996) Robust control of infinite-dimensional systems: frequency domain methods. Lecture notes in control and information sciences, vol 209. Springer, London

Gu G, Khargonekar PP, Lee EB (1989) Approximation of infinite-dimensional systems. IEEE Trans Autom Control 34:610-618

Gumussoy S (2011) Coprime-inner/outer factorization of SISO time-delay systems and FIR structure of their optimal $\mathcal{H}_{\infty}$ controllers. Int J Robust Nonlinear Control 22:981-998

Gumussoy S, Michiels W (2011) Fixed-order H-infinity control for interconnected systems using delay differential algebraic equations. SIAM J Control Optim 49(5):2212-2238

Gumussoy S, Özbay H (2004) On the mixed sensitivity minimization for systems with infinitely many unstable modes. Syst Control Lett 53:211-216

Meinsma G, Mirkin L, Zhong Q-C (2002) Control of systems with I/O delay via reduction to a one-block problem. IEEE Trans Autom Control 47:1890-1895

Monje CA, Chen YQ, Vinagre BM, Xue D, Feliu V (2010) Fractional-order systems and controls, fundamentals and applications. Springer, London

Morris KA (2001) $\mathcal{H}_{\infty}$-output feedback of infinitedimensional systems via approximation. Syst Control Lett 44:211-217

Özbay H (2000) Introduction to feedback control theory. CRC Press LLC, Boca Raton

Özbay H (2010) Stable $\mathcal{H}_{\infty}$ controller design for systems with time delays. In: Willems JC et al (eds) Perspectives in mathematical system theory, control, and signal processing. Lecture notes in control and information sciences, vol 398. Springer, Berlin/Heidelberg, pp 105-113

Sipahi R, Niculescu S-I, Abdallah CT, Michiels W, Gu K (2011) Stability and stabilization of systems with time delay. IEEE Control Syst Mag 31(1):38-65

Stein G (2003) Respect the unstable. IEEE Control Syst Mag 23(4):12-25

van Keulen B (1993) $\mathcal{H}_{\infty}$-control for distributed parameter systems: a state space approach. Birkhäuser, Boston

Wakaiki M, Yamamoto Y, Özbay H (2013) Stable controllers for robust stabilization of systems with infinitely many unstable poles. Syst Control Lett 62:511-516 
Zhong QC (2006) Robust control of time-delay systems. Springer, London

Zhou K, Doyle JC, Glover K (1996) Robust and optimal control. Prentice-Hall, Upper Saddle River

\section{Robust Fault Diagnosis and Control}

\author{
Steven X. Ding \\ University of Duisburg-Essen, Duisburg, \\ Germany
}

\section{Abstract}

Aiming at increasing system reliability and availability, integration of fault diagnosis into feedback control systems and integrated design of control and diagnosis receive considerable attention in research and industrial applications. In the framework of robust control, integrated diagnosis and control systems are designed to meet the demand for system robustness. The core of such systems is an observer that delivers needed information for a robust fault detection and feedback control.

\section{Keywords}

Observer-based fault diagnosis and control; Residual generation

\section{Introduction}

Advanced automatic control systems are marked by the high integration degree of digital electronics, intelligent sensors, and actuators. In parallel to this development, a new trend of integrating model-based fault detection and isolation (FDI) into the control systems can be observed (Blanke et al. 2006; Ding 2013; Gertler 1998; Isermann 2006; Patton et al. 2000), which is strongly driven by the enhanced needs for system reliability and availability.

A critical issue surrounding the integration of a diagnostic module into a feedback control loop is the interaction between the control and diagnosis. Initiated by Nett et al. (1988), study on the integrated design of control and diagnosis has received much attention, both in the research and application domains. The original idea of the integrated design scheme proposed by Nett et al. (1988) is to manage the interactions between the control and diagnosis in an integrated manner (Ding 2009; Jacobson and Nett 1991).

Robustness is an essential performance for model-based control and diagnostic systems. In the control and diagnosis framework, robustness is often addressed in different context (Ding 2013) and thus calls for special attention in the integrated design of control and diagnostic systems. In their study on fault-tolerant controller architecture, Zhou and Ren (2001) have proposed to deal with the integrated design in the framework of the Youla parametrization of stabilization controllers (Zhou et al. 1996), which also builds the basis for achieving high robustness in an integrated control and diagnosis system. Below, we present the basic ideas and some representative schemes and methods for the integrated design of robust diagnosis and control systems.

\section{Plant Model and Factorization Technique}

Consider linear time invariant (LTI) systems given in the state space representation

$$
\begin{aligned}
\dot{x}(t) & =A x(t)+B u(t)+E_{d} d(t)+E_{f} f(t) \\
y(t) & =C x(t)+D u(t)+F_{d} d(t)+F_{f} f(t) \\
z(t) & =C_{z} x(t)+D_{z} u(t)
\end{aligned}
$$

where $x \in \mathcal{R}^{n}, y \in \mathcal{R}^{m}, u \in \mathcal{R}^{k_{u}}$ stand for the plant state, output, and input vectors, respectively. $z \in \mathcal{R}^{k_{z}}$ is the controlled output vector. $d \in \mathcal{R}^{k_{d}}, f \in \mathcal{R}^{k_{f}}$ denote disturbance and fault vectors, respectively. $A, B, C, D, C_{z}, D_{z}, E_{d}, E_{f}, F_{d}, F_{f}$ are known matrices of appropriate dimensions.

A transfer matrix $G(s)=D+C(s I-$ $A)^{-1} B$ with the minimal state space realization $(A, B, C, D)$ can be factorized into 